\title{
Detection of chromosome copy number alterations in metanephric adenomas by array comparative genomic hybridization
}

\author{
Chin-Chen $\operatorname{Pan}^{1,2}$ and Jonathan I Epstein ${ }^{3}$ \\ ${ }^{1}$ Department of Pathology, Taipei Veterans General Hospital, Taipei, Taiwan; ${ }^{2}$ Department of Pathology, \\ National Yang-Ming University, Taipei, Taiwan and ${ }^{3}$ Department of Pathology, The Johns Hopkins University \\ School of Medicine, The James Buchanan Brady Urological Institute, The Johns Hopkins Hospital,
} Baltimore, MD, USA

\begin{abstract}
Metanephric adenoma is a rare benign renal tumor typically found in adults. Previous cytogenetic analyses, including karyotyping, fluorescence in situ hybridization (FISH), and comparative genomic hybridization, have yielded conflicting results regarding the somatic genetic aberrations of these tumors. In this study, we investigated the genomic profile of nine cases of metanephric adenoma using array comparative genomic hybridization. Two cases revealed multiple chromosomal gains and losses. Three cases showed sporadic chromosomal imbalances involving no more than three chromosomes. Four cases showed normal chromosome copy numbers. The gain of chromosome 19 was the most common finding (five cases), and FISH using 19p and 19q telomeric probes further confirmed this finding. We did not observe consistent gains of chromosomes 7 and 17, which are common in papillary renal cell carcinoma, neither did we find chromosomal alterations frequently present in Wilms' tumors, including chromosome gains of 1q, 7q, and 12, and losses of $11 p$ and 16q. Our series demonstrates that the genetic profile of metanephric adenoma is fundamentally distinct from those of papillary renal cell carcinoma and Wilms' tumor.
\end{abstract}

Modern Pathology (2010) 23, 1634-1640; doi:10.1038/modpathol.2010.162; published online 27 August 2010

Keywords: array comparative genomic hybridization; fluorescence in situ hybridization; metanephric adenoma

On the basis of the WHO classification of renal tumors, metanephric tumors comprise metanephric adenomas, metanephric adenofibromas, and metanephric stromal tumors, ${ }^{1}$ with each of these having its own characteristic clinicopathological presentation. Metanephric adenoma is defined as a highly cellular epithelial tumor composed of small, uniform, and embryonic-appearing cells. The tumor occurs most commonly in patients in their 50s and 60 s, with a female preponderance. Although the vast majority of metanephric adenomas behave in a benign fashion, cases of regional lymph node metastasis and sarcomatoid changes have been individually reported. ${ }^{2-4}$

Correspondence: Dr C-C Pan, MD, Department of Pathology, Taipei Veterans General Hospital, No. 201, Shi-Pai Road, Section2, Taipei 11217, Taiwan.

E-mail: ccpan@vghtpe.gov.tw

Received 21 April 2010; revised 28 June 2010; accepted 11 July 2010; published online 27 August 2010
The morphological and immunohistochemical characteristics of metanephric adenomas have been well described. ${ }^{5-7}$ However, cytogenetic or molecular genetic data about this rare tumor are limited and have mostly been reported for single cases. Previous karyotyping of a total of five cases revealed negative findings, with the exception of a dual balanced translocation of $\mathrm{t}(1 ; 22)(\mathrm{q} 22 ; 13)$ and $\mathrm{t}(15 ; 16)(\mathrm{q} 21 ; \mathrm{p} 13)$ in one case. ${ }^{5,8-12}$ One fluorescence in situ hybridization (FISH) study indicated gain of chromosomes 7 and 17 and loss of Y chromosome-the cytogenetic markers of papillary renal cell carcinoma-in 8 of 11 metanephric adenomas. ${ }^{13}$ However, subsequent FISH analyses revealed normal copy numbers for chromosomes 7 and $17 .^{4,8,11,14,15} \mathrm{~A}$ deletion in chromosome $2 \mathrm{p}$ was the only genetic abnormality described in one tumor, and a tumor suppressor gene region on $2 \mathrm{p} 13$ was delineated. ${ }^{16,17}$ Most recently, a study employing array comparative genomic hybridization (aCGH) reported no copy number changes in six cases of metanephric adenoma. $^{18}$ 
In this study, we aimed to delineate the chromosomal profile of metanephric adenoma using aCGH. Possible alterations were further validated by FISH analysis using appropriate probes. The series recruited five cases from the pathology consult file of The Johns Hopkins Hospital and four patients from the surgical pathology file of Taipei Veterans General Hospital. The study was approved by the institutional review board of Taipei Veterans General Hospital (IRB 98-01-04A).

\section{Materials and methods}

\section{Sample Collection}

Initially we collected tumor samples from of a total of 12 patients. The samples were fixed in formalin and embedded in paraffin. Nine of these samples had DNA of sufficient quality and were used for subsequent analysis. The patients from whom these samples were obtained consisted of eight women and one man, with ages ranging from 40 to 61 (mean, 49) years. The tumors exhibited typical morphology (Figure 1). The paraffin blocks were punctured at the most suitable site for DNA extraction corresponding to the most cellular area of the tumor based on hematoxylin and eosin-stained slides.

An immunohistochemical assay including WT1 (1:50, Dako, Glostrup, Denmark), CD57 (1:50, Becton-Dickinson, San Jose, CA, USA), AE1/3 (1:30, Zymed, San Francisco, CA, USA), epithelial membrane antigen (1:40, Dako), keratin 7 (1:50, Dako), and $\alpha$-methylacyl-CoA racemase (1:100, Zymed) was performed.

One sample from a solid variant of papillary renal cell carcinoma was used for comparison, as solid variant papillary renal cell carcinoma is a major differential diagnosis of metanephric adenoma.

\section{DNA Extraction}

Fomalin-fixed, paraffin-embedded tissues were deparaffinized using xylene and washed in ethanol. A $1 \mathrm{M}$ solution of sodium thiocyanate was added to the dry pellet and incubated at $37^{\circ} \mathrm{C}$ overnight to remove cross-links. After removal of the sodium thiocyanate, a QIAamp (Qiagen, Foster City, CA, USA) extraction procedure was performed according to the manufacturer's instruction. After adsorbed onto a QIAamp silica membrane, the DNA was washed and eluted. The quantity (OD $260 \mathrm{~nm}$ ) and quality (OD $260 \mathrm{~nm} / \mathrm{OD} 280 \mathrm{~nm}$ ) of DNA in the obtained solution was measured using an ND-1000 spectrophotometer (Nanodrop Technology, Wilmington, DE, USA). The integrity of the DNA was determined using an Agilent 2100 Bioanalyzer (Agilent Technologies, Santa Clara, CA, USA). DNA samples with a length profile showing a sufficient amount of single-strand DNA of over $500 \mathrm{bp}$ in length were used of aCGH analysis.

\section{aCGH Analysis}

Genomic DNA $(2 \mu \mathrm{g})$ isolated from the tissue samples and DNA from healthy tissue (Promega Corporation, Madison, WI, USA) were labeled with Cy5 and Cy3, respectively, using an Oligo aCGH Labeling Kit (Agilent Technologies). The Cy5labeled and Cy3-labeled samples were purified using Agilent-KREApure columns (Agilent Technologies). The appropriately purified and labeled samples were processed according to the manufacturer's protocol. Before hybridization to the array, the hybridization mixtures were denatured at $95^{\circ} \mathrm{C}$ for $3 \mathrm{~min}$ and incubated at $37^{\circ} \mathrm{C}$ for $30 \mathrm{~min}$, and the samples were then applied to the Agilent Genome CGH Microarray 105A (Agilent Technologies). Hybridization was carried out for $40 \mathrm{~h}$ at $65^{\circ} \mathrm{C}$. After
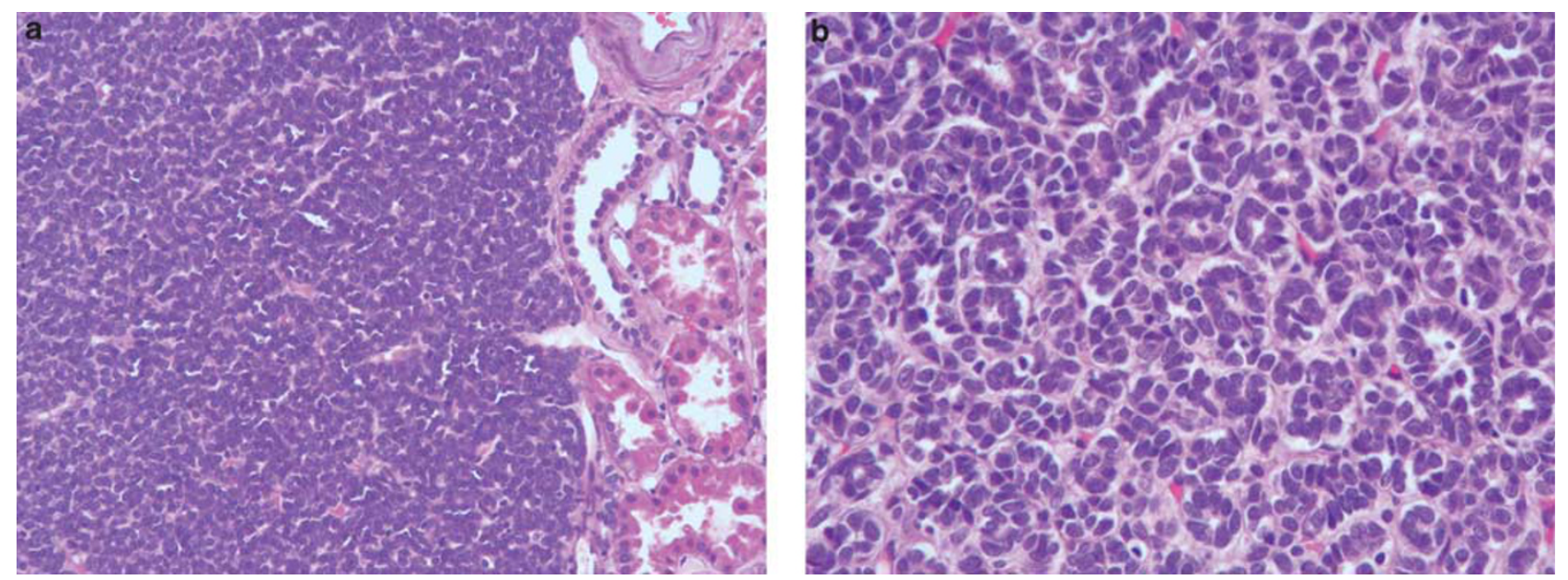

Figure 1 Metanephric adenoma is characterized by a well demarcated but nonencapsulated tumor (a) composed chiefly of hypercellular uniform cells in tubular and solid patterns (b). (hematoxylin and eosin, original magnification, $\mathbf{a}: \times 100, \mathbf{b}: \times 400$ ). 
washing, the slides were scanned on an Agilent microarray scanner. The images were analyzed using Feature Extraction software, version 10.5.1.1 (Agilent Technologies) and DNA Analytics software, version 4 (Agilent Technologies) using the ADM-2 algorithm with the following settings: $2 \mathrm{Mb}$ moving average; threshold, 6; filter, 3 probes; and 0.4 $\log 2$ ratio.

\section{FISH Analysis}

Sections $(5 \mu \mathrm{m})$ of the formalin-fixed paraffinembedded tissue blocks were mounted on siliconcoated glass slides, deparaffinized, and treated with pretreatment buffer (Zytovision, Bremerhaven, Germany) at $95^{\circ} \mathrm{C}$ for $15 \mathrm{~min}$. The FISH reaction was performed as previously described. ${ }^{19}$ FISH was performed with telomeric DNA probes for chromosome 19p (conjugated with Texas red) and chromosome 19q (conjugated with fluorescein isothiocyanate). FISH using centromeric probes for chromosome 7 (conjugated with Texas red) and chromosome 17 (conjugated with fluorescein isothiocyanate) was run in parallel. The probes were purchased from Cytocell (Cambridge, UK).
The images were acquired using a charge-coupled device camera together with the manufacturer's software program (Micrometrics 190CU; Accuscope, Commack, NY). The single-color images were merged using the Image-J program (http://rsbweb. nih.gov/ij/). Approximately 25-50 high-power fields were counted for each probe. The cutoff values for each probe were determined at mean values +3 s.d. of the signal percentages in the control renal tissue. Thus, gains were interpreted when the percentages of nuclei containing hyperdiploid signals exceeded $15 \%$.

\section{Results}

The alterations in copy number and immunohistochemical findings are listed in Table 1. Two cases (nos. 4 and 8) revealed multiple chromosomal imbalances. Three cases showed sporadic aberrations involving no more than three chromosomes (nos. 1, 2, and 7). The most common aberration was chromosome 19p amplification (five cases). Gain of chromosome 19q gain was less frequent (three cases). Four cases (nos. 3, 5, 6, and 9) showed no change in copy number. We did not observe

Table 1 Summary of chromosomal imbalances in metanephric adenoma

\begin{tabular}{|c|c|c|c|c|c|c|c|c|c|c|c|}
\hline \multirow[b]{2}{*}{ No. } & \multirow[b]{2}{*}{ Age } & \multirow[b]{2}{*}{ Sex } & \multicolumn{2}{|c|}{ Chromosomal imbalance } & \multicolumn{6}{|c|}{ Immunohistochemistry } & \multirow[b]{2}{*}{ Source } \\
\hline & & & Gain & Loss & $W T 1$ & $C D 57$ & $A E 1 / 3$ & $E M A$ & CK7 & $A M A C R$ & \\
\hline 1 & 61 & $\mathrm{~F}$ & 19p12-p13.3 & Nil & $3+$ & $2+$ & 0 & 0 & 0 & 0 & $\mathrm{~J}$ \\
\hline 2 & 42 & M & $\begin{array}{l}\text { 11q12.2-q13.4 } \\
19 \mathrm{p} 12-\mathrm{p} 13.3\end{array}$ & Nil & $3+$ & $3+$ & 0 & 0 & 0 & 0 & $J$ \\
\hline 3 & 59 & $\mathrm{~F}$ & Nil & Nil & $3+$ & $2+$ & $1+$ & 0 & 0 & 0 & $\mathrm{~J}$ \\
\hline 4 & 53 & $\mathrm{~F}$ & $\begin{array}{l}1 \mathrm{p} 33-\mathrm{p} 36.33 \\
6 \mathrm{p} 21.1-\mathrm{p} 22.1 \\
9 \mathrm{q} 33.3-\mathrm{q} 34.3 \\
11 \mathrm{q} 12.2-\mathrm{q} 13.4 \\
12 \mathrm{q} 13.11-\mathrm{q} 14.1 \\
16 \mathrm{p} 11.2-\mathrm{p} 13.3 \\
17 \mathrm{p} 13.1-\mathrm{p} 13.3, \mathrm{q} 12-\mathrm{q} 25.3 \\
19 \mathrm{p} 13.11-\mathrm{p} 13.3, \mathrm{q} 13.11-\mathrm{q} 13.43 \\
22 \mathrm{q} 11.21-\mathrm{q} 13.31\end{array}$ & $\begin{array}{l}\text { 1p11.3-p13.3, 1q25.3-q32.1 } \\
\text { 3p11.1-p13 } \\
\text { 4q12-q35.1 } \\
\text { 5p15.33-q31.1 } \\
\text { 7q11.23-q21.3, q31.1-q31.33 } \\
\text { 8q11.21-q24.3 } \\
\text { 9p13.3-p24.1 } \\
\text { 10q11.23-q21.3 } \\
\text { 12q15-q21.33 } \\
\text { 13q14.3-q32.1 } \\
\text { 14q13.3-q22.1 } \\
\text { 18q11.2-q23 } \\
21 q 11.2-q 22.11 \\
\text { Xp22.33-q28 }\end{array}$ & $3+$ & $2+$ & 0 & 0 & 0 & 0 & J \\
\hline 5 & 40 & $\mathrm{~F}$ & Nil & Nil & $3+$ & $3+$ & $1+$ & 0 & 0 & 0 & $\mathrm{~J}$ \\
\hline 6 & 52 & $\mathrm{~F}$ & Nil & Nil & $3+$ & $3+$ & 0 & 0 & 0 & 0 & $\mathrm{~T}$ \\
\hline 7 & 45 & $\mathrm{~F}$ & $\begin{array}{l}\text { 11q12.2-q13.3 } \\
\text { 19p13.11-p13.3 } \\
\text { 19q13.11-q13.33 }\end{array}$ & $\mathrm{Xq11.1-Xq28}$ & $3+$ & $2+$ & 0 & 0 & 0 & 0 & $\mathrm{~T}$ \\
\hline 8 & 42 & $\mathrm{~F}$ & $\begin{array}{l}9 \mathrm{q} 33.3-\mathrm{q} 34.3 \\
11 \mathrm{q} 12.2-\mathrm{q} 13.4 \\
17 \mathrm{p} 13.1-\mathrm{p} 13.3 \\
17 \mathrm{q} 12-\mathrm{q} 21.33,17 \mathrm{q} 25.1-\mathrm{q} 25.3 \\
19 \mathrm{p} 13.11-\mathrm{p} 13.3, \mathrm{q} 13.11-\mathrm{q} 13.43 \\
22 \mathrm{q} 11.1-\mathrm{q} 13.33\end{array}$ & $\begin{array}{l}3 \mathrm{p} 11.1-\mathrm{p} 13 \\
4 \mathrm{q} 12-\mathrm{q} 35.1 \\
7 \mathrm{q} 21.11-\mathrm{q} 31.33 \\
12 \mathrm{q} 14.1-\mathrm{q} 22 \\
21 \mathrm{q} 11.2-\mathrm{q} 22.11\end{array}$ & $3+$ & $3+$ & 0 & 0 & 0 & 0 & $\mathrm{~T}$ \\
\hline 9 & 49 & $\mathrm{~F}$ & Nil & Nil & $3+$ & $3+$ & 0 & 0 & 0 & 0 & $\mathrm{~T}$ \\
\hline
\end{tabular}

Abbreviations: 0, negative; 1+, weak; 2+, moderate; 3+, strong; AMACR, $\alpha$-methylacyl-CoA racemase; EMA, epithelial membrane antigen; J, The Johns-Hopkins Hospital; T, Taipei Veterans General Hospital. 
consistent chromosome gains on chromosomes 7 and 17 , as was the characteristic of papillary renal cell carcinoma. The two cases (nos. 4 and 8) showing partial gains of chromosome 17 also harbored other random chromosomal gains and losses. Except for one case showing partial gain of chromosome 12q (case 4, +12q13.11-q14.1), we did not observe chromosomal changes commonly seen in Wilms' tumors, such as $+1 \mathrm{q},+7 \mathrm{q},-11 \mathrm{p},+12$, and $-16 q$. Immunohistochemically, all cases were positive for WT1 (9/9) and $\operatorname{CD57}$ (9/9); two cases were weakly positive for cytokeratin AE1/3 (2/9); and all were negative for epithelial membrane antigen (0/9), cytokeratin $7(0 / 9)$, and $\alpha$-methylacylCoA racemase $(0 / 9)$.

FISH analysis confirmed the gains of chromosome 19 in cases observed to have chromosome 19 amplification based on aCGH. Cases without gain of chromosome 19 based on aCGH also showed normal copy number in the FISH analysis. The signals of the centromeric probes for chromosomes 7 and 17 were within normal ranges in all cases. The results of aCGH and FISH analyses are depicted in Figure 2.

The solid variant papillary renal cell carcinoma demonstrated gains of chromosomes 7 and 17 (Figure 3). The FISH analysis performed on this case also revealed trisomy of chromosomes 7 and 17. The tumor was immunoreactive for AE1/3, epithelial membrane antigen, keratin 7 , and $\alpha$-methylacylCoA racemase, but negative for WT1 and CD57. The genetic and immunohistochemical pattern was characteristic of papillary renal cell carcinoma.

Four patients had available follow-up records. Two of these patients showed multiple genetic imbalances (nos. 7 and 8) and were alive without disease for 8 and 2 years, respectively. The other two patients, who had normal chromosomal profiles (nos. 6 and 9), were alive without disease for 15 and 6 years, respectively.

\section{Discussion}

Previous reports concerning the cytogenetic analyses of metanephric adenomas have been conflicting. The findings in the series of Brown et al, who reported trisomies of chromosomes 7 and 17 and loss of Y chromosome in 8 of 11 cases diagnosed as metanephric adenoma, were not reproduced in later investigations. The later series all showed contrary results..$^{4,8,11,13-15}$ Our aCGH analysis, as well as that reported by Szponar et $a{ }^{18}{ }^{18}$ also failed to identify gains of chromosomes 7 and 17. Given that papillary renal cell carcinoma may exhibit a solid growth pattern, it is highly plausible that the presumed metanephric adenomas with trisomies of chromosomes 7 and 17 in the series of Brown et al actually represented solid variant of papillary renal cell carcinoma. $^{20}$ This speculation was further supported by the characteristic gains of chromosomes
7 and 17 in the solid variant of papillary renal cell carcinoma assayed in this study.

The genetic profile of metanephric adenoma differs significantly with that of Wilms' tumor. None of our cases exhibited deletion of chromosome 11p, in which the WT1 and WT2 genes are located. ${ }^{21,22}$ Other common genetic imbalances such as +12 and $+1 q /-16 q$ were also not found in our cases of metanephric adenoma. ${ }^{23-26}$ We did not observe amplification of $7 \mathrm{q}$ corresponding to isochromosome $7 q$ observed in adult Wilms' tumors. ${ }^{27}$ Despite some phenotypic overlap between metanephric adenoma and predominantly epithelial Wilms' tumor (immunoreactivity for WT-1, for example), there was no evidence to indicate any association between the two neoplasms from the molecular genetic point of view.

Another previously reported aberration in metanephric adenoma is the partial deletion of chromosome $2 \mathrm{p}$, as demonstrated by karyotyping, conventional CGH, and microsatellite allotyping. ${ }^{16,17}$ We did not observe any alteration on chromosome 2p13. Nevertheless, owing to the resolution of aCGH, we could not exclude the possibility of a small deletion that might be below the detection limit of aCGH. aCGH is also unable to detect balanced translocations, as there is no dosage change in the involved genome.

Around half of our cases revealed euploidy, and only two of nine cases revealed multiple chromosomal dosage changes. Most previous cytogenetic studies have revealed a paucity of genetic alterations. Using microsatellite analyses, Pesti et al ${ }^{17}$ demonstrated sporadic allelic imbalances involving chromosomes 2p, 7, 8p, 12q, 16q, and 20q in $18-56 \%$ of cases. Factors including sensitivity of the methodology, quality of the DNA, efficiency of extraction, and tumor proportion may influence the detection threshold of the employed assays. These low-level genetic alterations are compatible with the benign neoplastic nature of metanephric adenoma, which is expected to be genetically slightly more variable than normal cells, yet not as unstable as malignant tumors. It would be of interest to determine whether metanephric adenomas harboring more genetic imbalances behave differently from those without observable genetic changes. We did not find any remarkable difference regarding the histological and immunohistochemical features between samples with and without genetic aberrations, as all of the patients who we recruited had typical metanephric adenomas with uniformly benign courses. Given that metanephric adenomas generally display benign biological behavior and only a limited number of cases have been studied molecularly, the significance of these positive findings are not evident at present.

We found frequent gain of chromosome 19, which has not been previously reported in metanephric adenoma, and we confirmed this finding with FISH. However, gain of chromosome 19 appears to be a 

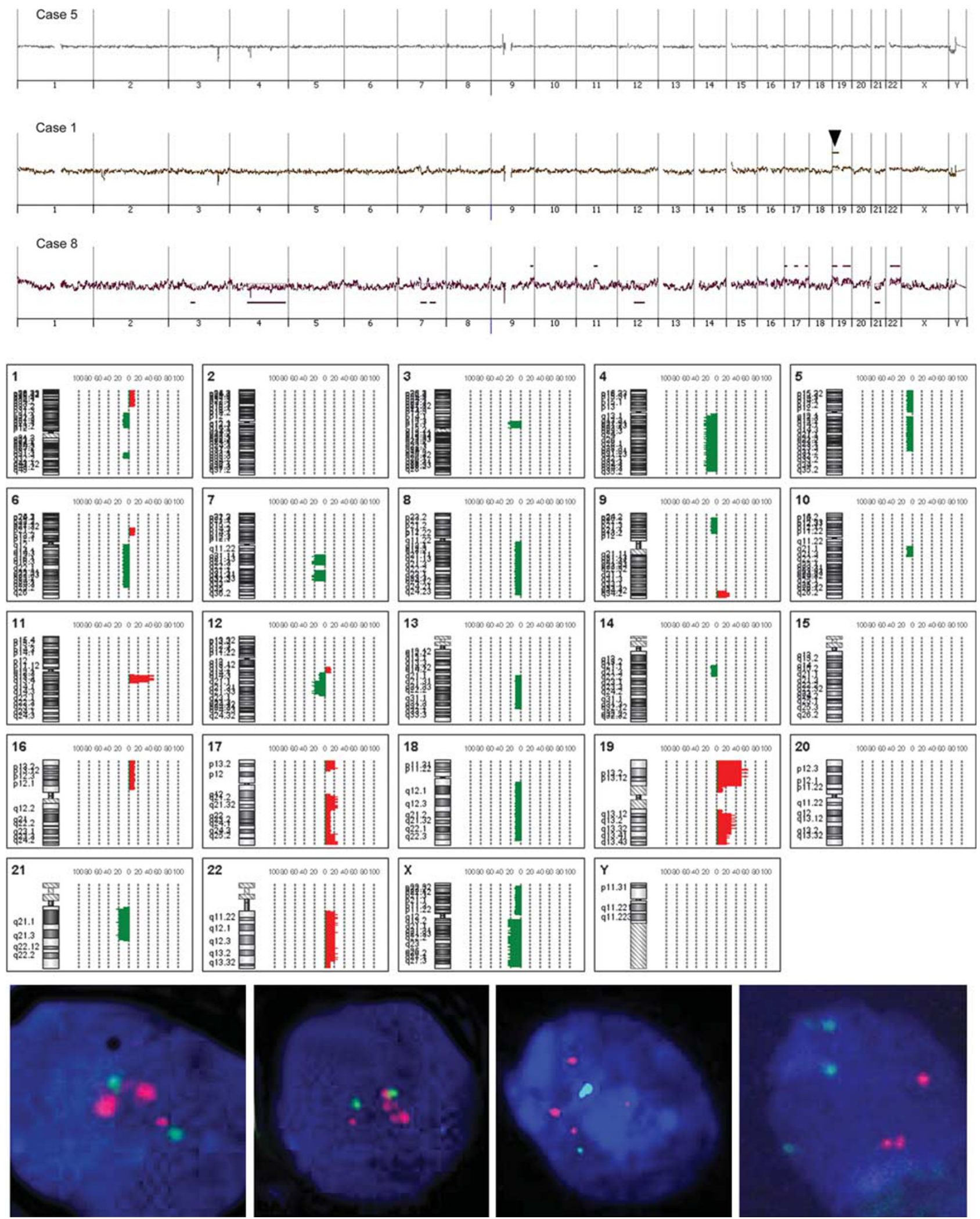

Figure 2 Top: array comparative genomic hybridization profiles of three metanephric adenomas. Case 5: no alteration; case 1: single gain on 19p (arrowhead); case 8: multiple aberrations. Center: accumulative penetrance plot of chromosomal imbalances of nine cases showing gains of chromosome 19 as the most common abnormality. Red bars indicate gains. Green bars indicate losses. Bottom: fluorescence in situ hybridization revealed increased copy number of chromosomes 19p (red) or 19q (green) using telomeric probes. 


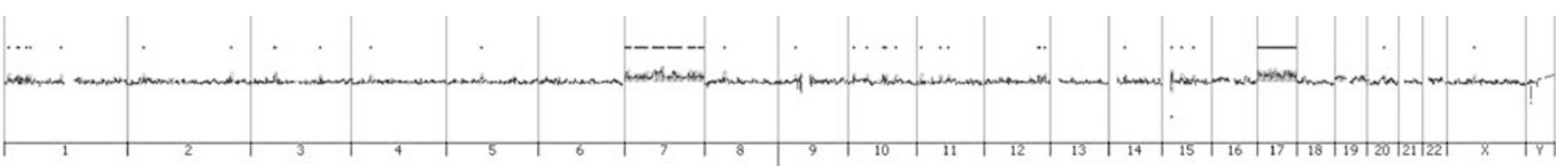

Figure 3 Array comparative genomic hybridization analysis of one solid variant of papillary renal carcinoma showing gains of chromosomes 7 and 17.

nonspecific finding, occasionally present in several different kinds of neoplasms. ${ }^{28-32}$ The presence of gains of chromosome 19 in metanephric adenomas implies the existence of a yet unrecognized oncogene in this region that may have a role in the tumorigenesis of this neoplasm.

In conclusion, we performed aCGH on nine metanephric adenomas and identified chromosomal imbalances in five cases, among which gain of chromosome 19 was the most common abnormality. From the genetic perspective, metanephric adenoma is a distinct entity despite certain morphological and immunophenotypic similarities to those of papillary renal cell carcinoma and Wilms' tumor.

\section{Acknowledgement}

This work was supported by Grants V96C1-031 and V98C1-097 from the Taipei Veterans General Hospital.

\section{Disclosure/conflict of interest}

The authors declare no conflict of interest.

\section{References}

1 Eble JN, Sauter G, Epstein JI, et al. World Health Organization Classification of Tumours. Pathology and Genetics of Tumours of the Urinary System and Male Genital Organs. IARC Press: Lyon, 2004.

2 Drut R, Drut RM, Ortolani C. Metastatic metanephric adenoma with foci of papillary carcinoma in a child: a combined histologic, immunohistochemical, and FISH study. Int J Surg Pathol 2001;9:241-247.

3 Picken MM, Curry JL, Lindgren V, et al. Metanephric adenosarcoma in a young adult: morphologic, immunophenotypic, ultrastructural, and fluorescence in situ hybridization analyses: a case report and review of the literature. Am J Surg Pathol 2001;25:1451-1457.

4 Renshaw AA, Freyer DR, Hammers YA. Metastatic metanephric adenoma in a child. Am J Surg Pathol 2000;24:570-574.

5 Jones EC, Pins M, Dickersin GR, et al. Metanephric adenoma of the kidney. A clinicopathological, immunohistochemical, flow cytometric, cytogenetic, and electron microscopic study of seven cases. Am J Surg Pathol 1995;19:615-626.

6 Muir TE, Cheville JC, Lager DJ. Metanephric adenoma, nephrogenic rests, and Wilms' tumor: a histologic and immunophenotypic comparison. Am J Surg Pathol 2001;25:1290-1296.
7 Olgac S, Hutchinson B, Tickoo SK, et al. Alphamethylacyl-CoA racemase as a marker in the differential diagnosis of metanephric adenoma. Mod Pathol 2006;19:218-224.

8 Burger M, Junker K, Denzinger S, et al. Metanephric adenoma of the kidney: a clinico-pathological and molecular study of two cases. J Clin Pathol 2007; 60:832-833.

9 Gatalica Z, Grujic S, Kovatich A, et al. Metanephric adenoma: histology, immunophenotype, cytogenetics, ultrastructure. Mod Pathol 1996;9:329-333.

10 Granter SR, Fletcher JA, Renshaw AA. Cytologic and cytogenetic analysis of metanephric adenoma of the kidney: a report of two cases. Am J Clin Pathol 1997; 108:544-549.

11 Tsuji M, Murakami Y, Kanayama H, et al. A case of renal metanephric adenoma: histologic, immunohistochemical and cytogenetic analyses. Int J Urol 1999;6: 203-207.

12 Lerut E, Roskams T, Joniau S, et al. Metanephric adenoma during pregnancy: clinical presentation, histology, and cytogenetics. Hum Pathol 2006;37: 1227-1232.

13 Brown JA, Anderl KL, Borell TJ, et al. Simultaneous chromosome 7 and 17 gain and sex chromosome loss provide evidence that renal metanephric adenoma is related to papillary renal cell carcinoma. J Urol 1997; 158:370-374.

14 Brunelli M, Eble JN, Zhang S, et al. Metanephric adenoma lacks the gains of chromosomes 7 and 17 and loss of $\mathrm{Y}$ that are typical of papillary renal cell carcinoma and papillary adenoma. Mod Pathol 2003; 16:1060-1063.

15 Renshaw AA, Maurici D, Fletcher JA. Cytologic and fluorescence in situ hybridization (FISH) examination of metanephric adenoma. Diagn Cytopathol 1997;16: 107-111.

16 Stumm M, Koch A, Wieacker PF, et al. Partial monosomy $2 p$ as the single chromosomal anomaly in a case of renal metanephric adenoma. Cancer Genet Cytogenet 1999;115:82-85.

17 Pesti T, Sukosd F, Jones EC, et al. Mapping a tumor suppressor gene to chromosome 2p13 in metanephric adenoma by microsatellite allelotyping. Hum Pathol 2001;32:101-104.

18 Szponar A, Yusenko MV, Kovacs G. High-resolution array CGH of metanephric adenomas: lack of DNA copy number changes. Histopathology 2010;56: 212-216.

19 Pan CC, Chen YJ, Chang LC, et al. Immunohistochemical and molecular genetic profiling of acquired cystic disease-associated renal cell carcinoma. Histopathology 2009;55:145-153.

20 Renshaw AA, Zhang H, Corless CL, et al. Solid variants of papillary (chromophil) renal cell carcinoma: clinicopathologic and genetic features. Am J Surg Pathol 1997;21:1203-1209. 
21 Gessler M, Poustka A, Cavenee W, et al. Homozygous deletion in Wilms tumours of a zinc-finger gene identified by chromosome jumping. Nature 1990;343: 774-778.

22 Ping AJ, Reeve AE, Law DJ, et al. Genetic linkage of Beckwith-Wiedemann syndrome to 11p15. Am J Hum Genet 1989;44:720-723.

23 Hoglund M, Gisselsson D, Hansen GB, et al. Wilms tumors develop through two distinct karyotypic pathways. Cancer Genet Cytogenet 2004;150:9-15.

24 Natrajan R, Williams RD, Hing SN, et al. Array CGH profiling of favourable histology Wilms tumours reveals novel gains and losses associated with relapse. J Pathol 2006;210:49-58.

25 Rassekh SR, Chan S, Harvard C, et al. Screening for submicroscopic chromosomal rearrangements in Wilms tumor using whole-genome microarrays. Cancer Genet Cytogenet 2008;182:84-94.

26 Vuononvirta R, Sebire NJ, Dallosso AR, et al. Perilobar nephrogenic rests are nonobligate molecular genetic precursor lesions of insulin-like growth factor-IIassociated Wilms tumors. Clin Cancer Res 2008;14: 7635-7644.
27 Rubin BP, Pins MR, Nielsen GP, et al. Isochromosome $7 \mathrm{q}$ in adult Wilms' tumors: diagnostic and pathogenetic implications. Am J Surg Pathol 2000;24: 1663-1669.

28 Carneiro A, Isinger A, Karlsson A, et al. Prognostic impact of array-based genomic profiles in esophageal squamous cell cancer. BMC Cancer 2008;8:98.

29 Choi YW, Choi JS, Zheng LT, et al. Comparative genomic hybridization array analysis and real time PCR reveals genomic alterations in squamous cell carcinomas of the lung. Lung Cancer 2007;55:43-51.

30 Jiang M, Li M, Fu X, et al. Simultaneously detection of genomic and expression alterations in prostate cancer using cDNA microarray. Prostate 2008;68: 1496-1509.

31 Mori H, Nagata M, Nishijima N, et al. Malignant pheochromocytoma in a young adult forming the structure simulating Homer Wright rosette: differentiation from neuroblastoma on repeating fluorescence in situ hybridization. Pathol Int 2008;58:518-523.

32 Poeaim S, Rerkamnuaychoke B, Jesdapatarakul S, et al. Chromosome alterations in colorectal cancer in Thai patients. Cancer Genet Cytogenet 2005;160:152-159. 